\title{
An Effective System for Content MRI Brain Image Retrieval using Angular Radial Transform
}

\author{
Abderrahim Khatabi \\ LMF, Department of Computer \\ Chouaib Doukkali University \\ Faculty of Science \\ B.P.20, 24000 \\ El Jadida, Morocco
}

\author{
Amal Tmiri \\ Laroseri, Department of Computer \\ Chouaib Doukkali University \\ Faculty of Science \\ B.P. 20,24000 \\ El Jadida, Morocco
}

\author{
Ahmed Serhir \\ LMF, Department of Computer \\ Chouaib Doukkali University \\ Faculty of Science \\ B.P. 20, 24000 \\ El Jadida, Morocco
}

\begin{abstract}
Nowadays, the growth of huge amount of medical image has become one of the most important clinical diagnosis components, Furthermore, there is an urgent need a system of Content Based Image Retrieval (CBIR) to obtain essential information such as type of image and extracting features of the image, such as color, shape and texture. This system is based on the image content that retrieves similar pathology involving magnetic resonance (MR) images of the medical database to assist the radiologist in the diagnosis of brain tumor; the shape recovery is the one of the top performers. Content-based image retrieval can also be used to locate brain tumors in medical images in large databases. In this paper, we propose a new method to build a new research system by the content of MRI images (CBIR) based on image characteristics, such as shape using the ART descriptor (angular radial transform) that is applied to reveal the characteristics of MR images. ART descriptor (angular radial transform) of shape is based Region adopted in MPEG-7 has the properties invariant to scale, rotation and robustness to noise, thanks to its properties we have used in this system. After the segmentation process, extracting the visual features of shape by calculating the coefficients of the ART and forming a second feature vector to be input to a support vector machine (SVM) for determining the presence tumor or not tumor followed by KNN (K-nearest neighbor) that retrieves the most similar images in the database. To provide faster image search.
\end{abstract}

\section{General Terms}

Content based image retrieval; Classification;

\section{Keywords}

ART descriptor (angular radial transform), Content based image retrieval (CBIR), magnetic resonance (MR) images, feature extraction, SVM, KNN.

\section{INTRODUCTION}

Brain tumor is one of the main causes of death among people all over the world. MRI is very important role to save the life. Doctors can miss the abnormality due to inexperience in the field of cancer[1] or tumor detection. This can be further categorized as benign or malignant. Early diagnosis can be increased the chances of survival using system of CBIR. System of Content Based Image Retrieval (CBIR) [2] that can help Doctors to do the diagnostic and therapeutic approach for these tumors and make the final decision to research the tumor and non-tumor.

Content Based Image Retrieval (CBIR) is becoming an important field with the advance of multimedia and imaging technology ever increasingly, describe the automatic retrieval of images [3] from a database, the technical CBIR [4] use these visual content such as color, texture and shape to represent and index images. Among them, the shapes contain rich of information than the color or the texture. However, research based on the contents of the shape is more difficult than based on color or texture because of the diversity of forms and the natural occurrence of the transformations of shape such as deformation, invariant to scale and orientation.

Representation and description of shape based region and contour can exploit the limits of shape information. Global approaches do not divide shape into sub-parts, usually a feature vector derived from the integral boundary is used to describe the shape. Various shape descriptors have been developed in the literature, which are broadly categorized into two types: contour-based and region-based shape descriptors. The angular Radial transformation (ART) is the shape descriptor based region on MPEG-7 [5] have rotational invariance properties [6-7]. It is defined on a disk of the unit in polar coordinates. The formulas of ART [8] are similar to moments of Zernike, but with a simpler form, while ART are observed to be the best among the region-based descriptors.

The query image bestowed to our criticism is the magnetic resonance brain images, which provides good disparity between the soft tissues of brain. in this paper the features of brain images extract by descriptor of shape based on region . Depending on those features, the feature vector is evaluated and given as the index for further classification of brain images under tumor or not tumor classes. The significant part of this diagnosis is to a support vector machine (SVM) [9] for classification [10] and determining the presence tumor or not tumor followed by KNN (K-nearest neighbor) [11] that retrieves the most similar images in the database for classifying [12] brain images according to its characteristics.

\section{Proposed System}

In CBIR, every image that is put away in the database has its features extracted and compared to the features of the query image. It includes two stages:

- Feature Extraction based on the visual content characteristics using descriptor of shape

- Feature Vector of MR images is input to a support vector machine (SVM) for determining the presence tumor or not tumor followed by KNN (K-nearest neighbor) that retrieves the most similar images from database that are similar to the query image Content-based image retrieval is an advanced method to search, navigate and browse large medical image. 


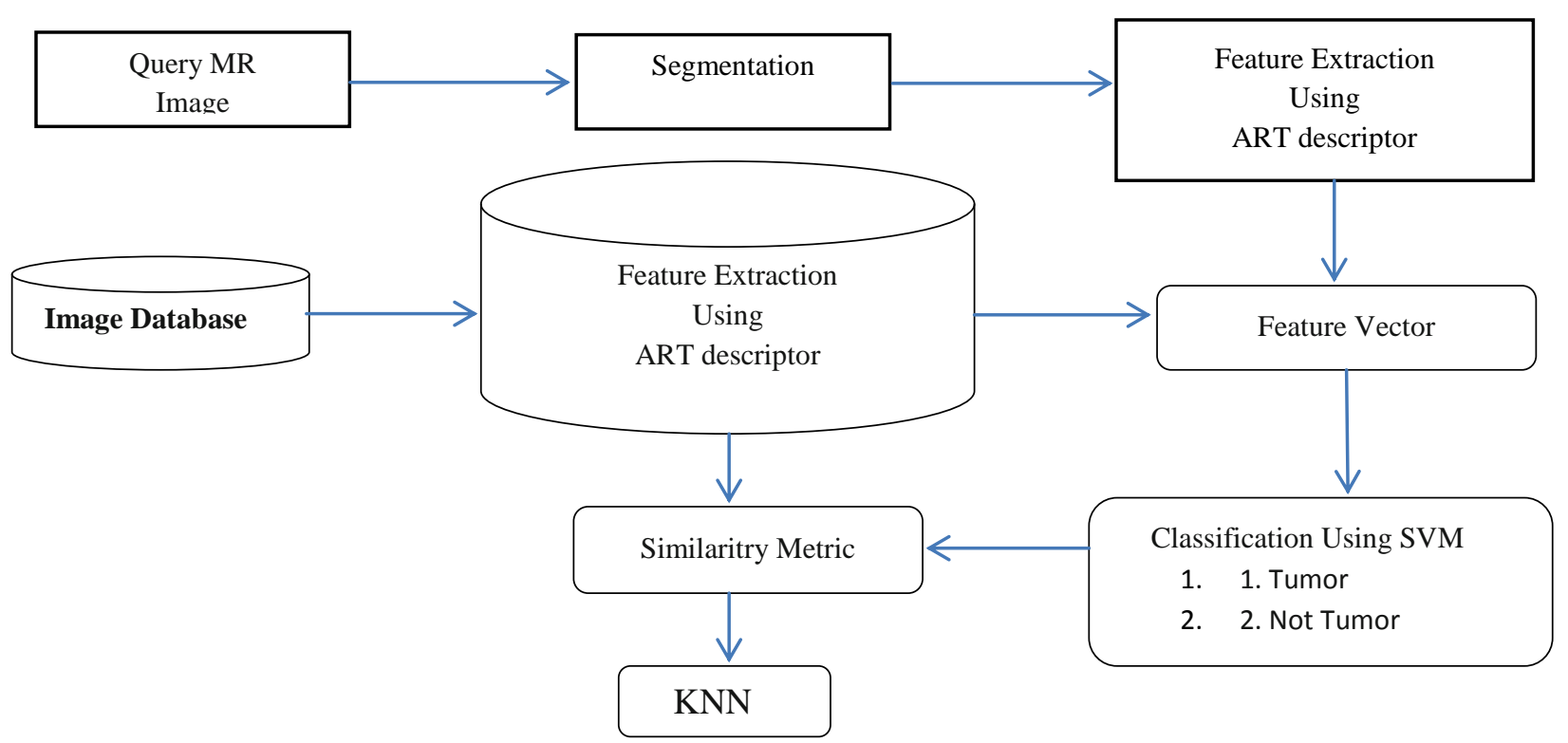

Fig1. Proposed CBIR system

\section{FEATURE EXTRACTION}

The first step in this methodology is to extract the query image features and database of MR images. The extraction of different features by descriptor of shape based region ART (angular radial transform) is one of the major stages in designing a reliable image retrieval system.

\section{ANGULAR RADIAL TRANSFORM}

\section{(ART)}

ART is a complex orthogonal unitary transform defined on a unit disk based on complex orthogonal sinusoidal basis functions in polar co-ordinates [13, 14, and 15]. The ART coefficients, of order $\mathrm{n}$ and $\mathrm{m}$, are defined by:

$$
F_{n m}=\int_{0}^{2 \pi} \int_{0}^{1} V_{n m}^{*}(r, \theta), f(r, \theta) r d r d \theta
$$

Where $f(r, \theta)$ is an image intensity function in polar coordinates and $V_{n m}^{*}(r, \theta)$ is a basis function, which is complex conjugate of $V_{n m}(r, \theta)$ defined in polar coordinates over a unit disk. These are expressed in a separable form of both radial and angular parts as follows:

$$
V_{n m}(r, \theta)=A_{m}(\theta) R_{n}(r)
$$

The indices $\mathrm{n}$ and $\mathrm{m}$ are nonnegative integers. The real valued radial polynomial and the angular basis function are defines as:

$$
R_{n}(r)= \begin{cases}1 & n=0 \\ 2 \cos (\pi n \rho) & n \neq 0\end{cases}
$$

$$
\text { And } \quad A_{m}(\theta)=\frac{1}{2 \pi} \exp (j m \theta) \quad \text { with } j=\sqrt{-1}
$$

The important properties of ART is the rotational invariance, the magnitude values of ART are unaffected and remain identical for image functions before and after rotation. The original image is represented by the intensity image function $f(r, \theta)$ having ART is rotated counterclockwise by angle $\alpha$; the transformed image function is $g(r, \theta)=f(r, \theta-\alpha)$. The ART coefficients of original and rotated images are $F_{n m}$ and $F_{n m}{ }^{r o t}=\exp (-j m \theta) F_{n m}$, the magnitude values are identical.

The default ART has 35 coefficients $(n=3, m=12)$.

The difference operation between two ART coefficient vectors use formed as:

Then the similarity

$$
\begin{array}{r}
\operatorname{Dist}_{q, s}^{m, n}=\sum_{i=1}^{m \times n}\left|x_{i}^{m, n}-y_{i}^{m, n}\right|_{\operatorname{Sim}_{A R T}}=\frac{1}{\operatorname{Dist}_{q, s}^{m \times n}}
\end{array}
$$

\subsection{Tumor Detection using ART and SVM}

Support Vector Machine (SVM) [16-17] is a discriminative classifier defined by a separating hyperplane that has been successfully used in a variety of pattern recognition tasks. We shall examine SVMs in the binary classification setting. Features vector of ART are used in training data form $\{\mathrm{X} 1 \ldots \mathrm{Xn}\}$ that are vectors in some space $X \in R^{n}$. We are also given their labels $\{\mathrm{L} 1 \ldots \mathrm{Ln}\}$, where $L i \in\{-1,1\}$. SVMs perform pattern recognition for two-class problem by making the decision boundary that has the maximum distance to the closest points in the training set.

If the data [18] are not linearly separable in the input space, SVMs allow us to project the data from the input space to a higher dimensional feature space via a Mercer kernel operator K [19] To allow for this kernels are used to non-linearly map the input data to a high-dimensional space In other words, we consider the set of classifiers of the form:

$$
u(x)=\sum_{i=1}^{N} \alpha_{i} K\left(X_{i}, X\right)
$$


The SVM training algorithms compute the values of that correspond to the maximal margin hyperplane in the feature space; kennels is the radial basis function (RBF) kernel [20]:

$$
K\left(\mathbf{x}_{\mathbf{i}}, \mathbf{x}_{\mathbf{j}}\right)=\exp \left(-\frac{\left\|\mathbf{x}_{\mathbf{i}}-\mathbf{x}_{\mathbf{j}}\right\|^{2}}{2 \sigma^{2}}\right)
$$

For two-class problems (tumor/non-tumor), the decision is made according to: when $f(X) \geq 0$ we classify $X$ as +1 (class 1 tumor); otherwise we classify $\mathrm{X}$ as -1 (class 2 - not tumor).

\subsection{Similarity Measures}

The purpose of a measure of similarity is to compare two regions (i.e. vectors); most measures were developed in the context of comparing feature vector of image. In our system we return the first images, whose distance from the query image is minimum [21]. In order to compute distance between query image $\mathrm{A}$ and database images $\mathrm{B}$, we use Euclidean distance, Mahalanobis distance, Manhattan distance.

The basis of many measures of similarity and dissimilarity is Euclidean distance. The distance between vectors A and B is defined as follows:

$$
D_{E}=\sqrt{\sum_{i=0}^{N-1}(A[i]-B[i])^{2}}
$$

\subsection{KNN (K_Nearest_Neighbors)}

$\mathrm{K}$ nearest neighbors is a supervised machine learning method, which stores all available cases and classify new cases based on a similarity measure. His Algorithm is based on classification[22] using a majority vote of its neighbors, with the case being assigned to the class most common amongst its $\mathrm{K}$ nearest neighbors measured by a Euclidean Distance.

\subsection{Performance Measure}

The performance is analyzed by computing the values of precision and recall.

Precision measures the ability of the system to retrieve number of relevant images retrieved to the total number of irrelevant and relevant records retrieved, while recall is the ratio of the number of relevant images retrieved [23] to the total number of relevant images in the database.

$$
\begin{aligned}
\text { Precision } & =\frac{\text { Total no. of Retrieval Relevant image }}{\text { Total no. of Retrieval image }} \\
\text { Recall } & =\frac{\text { Total no. of Retrieval Relevant image }}{\text { Total no. of Relevant image }}
\end{aligned}
$$

\section{EXPERIMENTAL RESULT}

We have mapped the resultant tumor image onto the original grayscale image for presentation purposes.

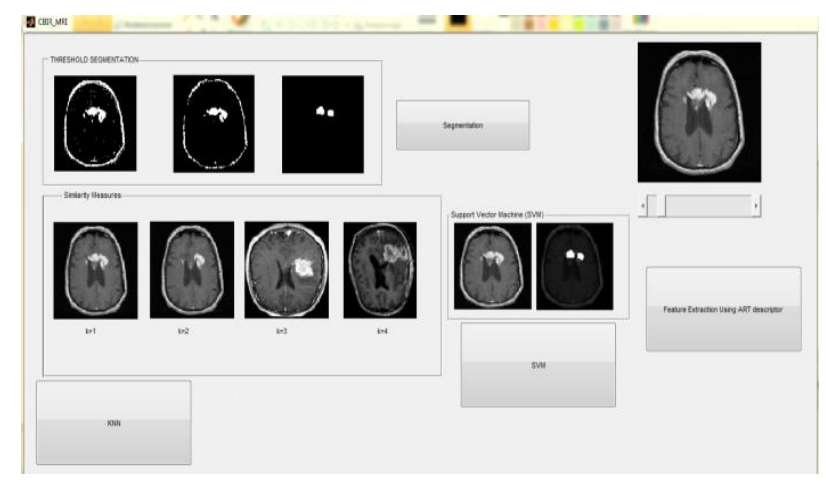

Fig2. Proposed system for CBIR of brain tumor

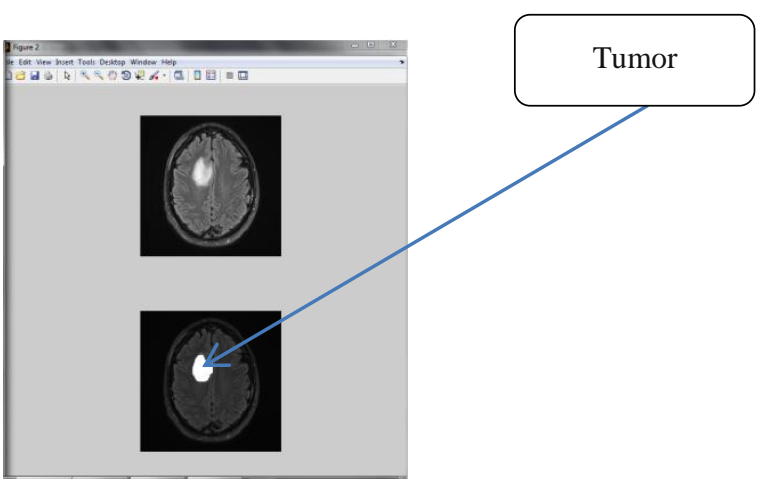

We propose new methodology for new system of Content Based Image Retrieval (CBIR) for MRI Brain Image [24] based on single query system. After segmentation the image is projected onto the feature space by extracting the shape features using Angular Radial Transform. Categorization of query image is done by SVM classifier. The database images are also classified in the same way for refining the searching. Euclidean Distance is utilized for searching the relevant images for the large database that helps radiologists to take the clinical decision for treatment. Our system is based on two different scenarios. In First scenario MRI image is classified in two different categories by SVM: Tumor and Not Tumor. In second scenario if any tumorous image is detected by classifier, the system detects the tumor in image. At last similar image is retrieved by KNN.

- $\quad$ analysis and results

Tab: Classification Accuracy comparison with other Methods

\begin{tabular}{|c|l|l|}
\hline No & \multicolumn{1}{|c|}{ Approach } & $\begin{array}{l}\text { Classification } \\
\text { Accuracy (\%) }\end{array}$ \\
\hline 1 & $\begin{array}{l}\text { Mina Rafi Nazari and E. } \\
\text { Fatemizadeh(October } \\
\text { 2010)GLCM+ PCA + SVM }\end{array}$ & $95 \%$ \\
\hline 2 & $\begin{array}{l}\text { DWT + PCA + ANN (EI- } \\
\text { Dahshan, Hosny, \& Salem, } \\
\text { 2010) }\end{array}$ & 98.33 \\
\hline Our system & ART+SVM+KNN & 98.45 \\
\hline
\end{tabular}

\section{FIGURES}

The results of performance analysis for precision and recall rates.

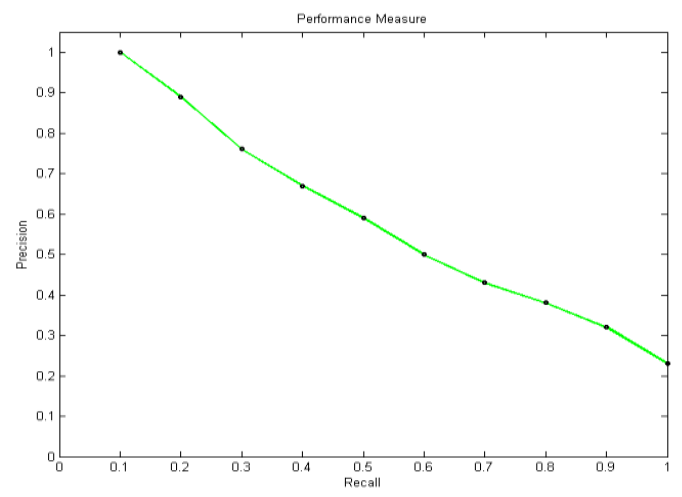




\section{CONCLUSION}

in this study we proposed method for an effective for build a system of CBIR that assists medical image diagnosis in clinical domain, this system based of Two different steps. In the First step, after segmentation and extraction by Angular Radial Transform Images are classified in tumor and and nottumorous category using SVM, in second scenario if any tumorous image detect, similarity measured by Euclidean Distance for searching the relevant images. At last similar image is retrieved by KNN

According to the experimental results, the proposed System is efficient for classification of the human brain MR database, and similar image.

\section{REFERENCES}

[1] H. Boussen, H. Bouzaiene, J. Ben Hassouna, A. Gamoudi, F. Benna, and K. Rahal, "Inflammatory breast cancer in Tunisia: reassessment of ,incidence and clinicopathological features". Semin Oncol. 2008, 35, pp.17-24.

[2] Mina Rafi Nazari and Emad Fatemizadeh, 'A CBIR System for Human Brain Magnetic Resonance Image', International Journal of Computer Applications (0975 8887) Volume 7- No.14, October 2010 Indexing.

[3] R.Guruvasuki, A. Josephine Pushpa Arasi, "MRI Brain Image retrieval using Multi Support Vector Machine Classifier",

[4] Hatice Cinar Akakin and Metin N. Gurcan, "ContentBased Microscopic Image Retrieval System for MultiImage Queries", IEEE Transaction on Information Technology in Biomedicine, Vol. 16, No. 4, pp 758-768, 2012.

[5] M. Bober, "MPEG-7 visual shape descriptors," IEEE Trans. Circuits Syst. Video Technol., vol. 11, pp. 716719, June. 2001

[6] Mokhtarian, F., S. Abbasi and J. Kittler, "Efficient and Robust Retrieval by Shape Content through Curvature Scale Space," Proc. International Workshop on Image Databases and Multimedia Search, pp. 35-42, Amsterdam, the Netherlands, 1996.

[7] M.-S. Choi and W. -Y. Kim, "The description and retrieval of a sequence of moving objects using a shape variation map," Pattern Recognition Letters, Vol. 25, pp.1369-1375, Sep. 2004

[8] S. Hwang and W. Kim, "Fast and Efficient Method for Computing ART," IEEE Transaction on Image processing, vol. 15, pp. 112-117, Jan. 2006.

[9] Nello Cristianini and John Shawe-Taylor, "An Introduction to Support Vector Machines and Other Kernel-based Learning Methods", Cambridge University
Press, 2000.Kharat, K.D., P.P. Kulkarni and M.B. Nagori, 2012. Brain tumor classification using neural network based methods. Int. J. Comput. Sci. Inform., 1:

[10] Amanatiadis, A., Kaburlasos, V. G., Gasteratos, A., \& Papadakis, S. E. (2011). Evaluation of shape descriptors for shape-based image retrieval. Image Processing, 5, 493-499.

[11] Forman, G. 2003. An extensive empirical study of feature selection metrics for text classification. J. Mach Learn. Res. 3 (Mar. 2003), 1289-1305.

[12] The Moving Picture Experts Group (MPEG), http://www.chiariglione.org/mpeg, 2009.12.01.

[13] Amanatiadis, A., Kaburlasos, V. G., Gasteratos, A., \& Papadakis, S. E. (2011). Evaluation of shape descriptors for shape-based image retrieval. Image Processing, 5, 493-499.

[14] Pooja, C. S. (2012). An effective image retrieval system using region and contour based features. In IJCA proceedings on international conference on recent advances and future trends in information technology (pp. 7-12).

[15] S. Haykin, Neural Networks - A Comprehensive Foundation, Prentice Hall Inc, 1999.

[16] M. Hearst, "Support vector machines", IEEE Intelligence Systems, pp. 18 - 28, July/August, 1998.

[17] J.P.Lewis, Tutorial on SVM, CGIT Lab, USC, 2004.

[18] S. Haykin, Neural Networks - A Comprehensive Foundation, Prentice Hall Inc, 1999

[19] Burges B. Scholkopf, editor, "Advances in Kernel Methods--Support Vector Learning”. MIT press, 1998.

[20] Pooja, C. S. (2012). An effective image retrieval system using region and contour based features. In IJCA proceedings on international conference on recent advances and future trends in information technology (pp. 7-12).

[21] Hatice Cinar Akakin and Metin N. Gurcan, "ContentBased Microscopic Image Retrieval System for MultiImage Queries", IEEE Transaction on Information Technology in Biomedicine, Vol. 16, No. 4, pp 758-768, 2012.

[22] Mohanpriya S., Vadivel M, "Automatic Retrieval of MRI Brain Image using Multiqueries System", IEEE Conference, pp 1099-1103, 2013.

[23] Neha Bhuptani and Bijal Talati, An Efficient Image Retrieval Technique using Shape Context Feature. International Journal of Computer Applications (0975 8887) Volume 98- No.1, July 2014. 